\title{
Bronchoscopic Drainage of Lung Abscesses Using a Pigtail Catheter
}

\author{
Avraham Unterman $^{\mathrm{a}, \mathrm{b}}$ Oren Fruchter ${ }^{\mathrm{b}, \mathrm{c}}$ Dror Rosengarten ${ }^{\mathrm{a}, \mathrm{b}}$ \\ Shimon Izhakian ${ }^{a, b}$ Nader Abdel-Rahman ${ }^{a, b}$ Mordechai R. Kramer ${ }^{a, b}$ \\ a Pulmonary Institute, Rabin Medical Center, Beilinson Campus, Petah Tikva, ${ }^{b}$ Sackler Faculty of Medicine, Tel Aviv \\ University, Tel Aviv, and 'Pulmonary Department, Wolfson Medical Center, Holon, Israel
}

\section{Keywords}

Bronchoscopy · Lung abscess · Drainage · Catheter ·

Treatment

\section{Abstract}

Background: Lung abscesses are commonly treated with antibiotics. However, some patients fail to respond and may require percutaneous catheter drainage or surgical intervention. Bronchoscopic drainage (BD) of lung abscesses emerged as a therapeutic alternative in selected patients. Objective: To describe our experience with 15 patients who underwent BD at our center during 2006-2016. Methods: Patients underwent flexible bronchoscopy. Under fluoroscopic guidance, a pigtail catheter was introduced into the abscess cavity, its correct position being confirmed by the injection of contrast medium. The catheter remained in place for a few days and was flushed repeatedly with antibiotics. Results: Fifteen patients (9 males; median age 59 years) underwent 16 BD procedures. A pigtail catheter was successfully inserted and pus was drained from the abscess cavity in 13 procedures (81\%) conducted in 12 patients, leading to rapid clinical improvement in 10 of them; resolution

\section{KARGER}

(c) 2016 S. Karger AG, Basel

E-Mail karger@karger.com

www.karger.com/res of fever occurred a median of 2 days (range <1-4) following pigtail insertion, and patients were discharged after 8 days (range 4-21). The pigtail catheter was extracted after a median of 4 days (range 2-6). Conclusion: BD of lung abscesses was achieved in 13 out of 16 procedures, leading to rapid improvement in the majority of patients. This work adds to the existing literature in establishing this procedure as an acceptable therapeutic alternative in selected patients who fail to respond to antibiotics, especially those with an airway obstruction or a fairly central lung abscess.

(c) 2016 S. Karger AG, Basel

\section{Introduction}

Lung abscesses are usually treated medically with antibiotics and postural drainage. Bronchoscopy is often performed in order to obtain cultures and to exclude and treat obstructive lesions [1]. However, some patients fail to respond to the above treatments and may require invasive abscess drainage [1,2]. Percutaneous catheter drainage (PCD), usually guided by computed tomography or ultrasound, is considered a less invasive alternative
Prof. Mordechai R. Kramer, MD, FCCP

Pulmonary Institute, Rabin Medical Center, Beilinson Campus Jabotinsky 39 St.

IL-49100 Petah Tikva (Israel)

E-Mail kramerm@ clalit.org.il 

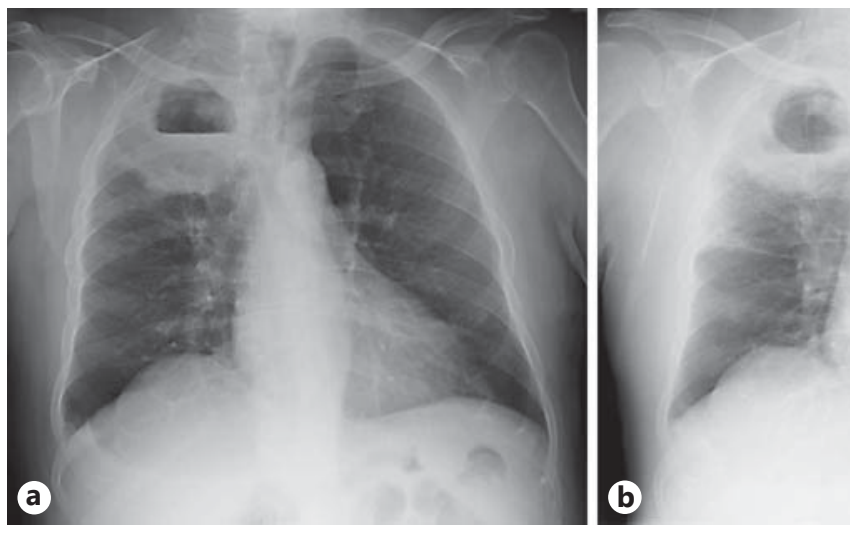

Fig. 1. a Chest radiograph of a 62-year-old man (patient 10, Table 1) with a right upper lobe abscess secondary to stage IV squamous cell carcinoma. The patient failed to respond to antibiotic treatment and underwent a bronchoscopic drainage procedure. b Chest radiograph taken $5 \mathrm{~h}$ following pigtail insertion showing the pigtail catheter tip inside the abscess, with a significant de-
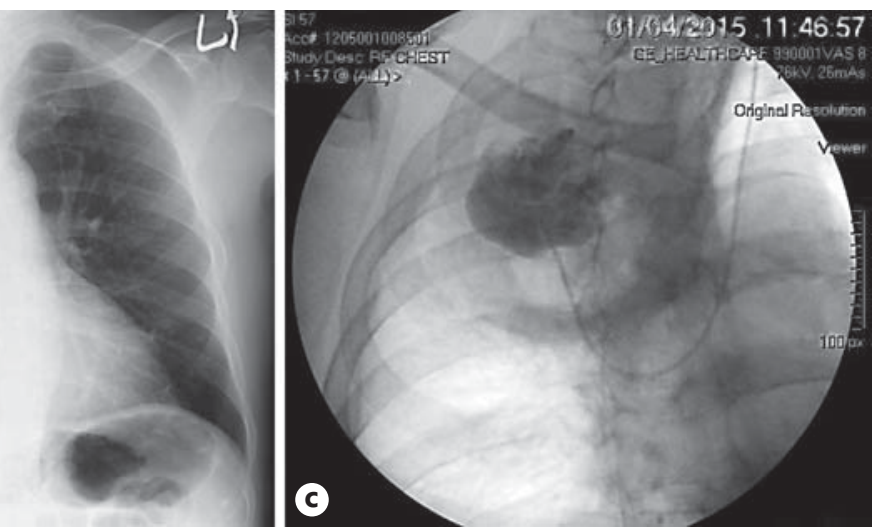

crease in fluid level. The patient quickly improved, fever resolved within 1 day, and the patient was discharged home 7 days following the procedure. c Fluoroscopic image taken during the procedure following pigtail insertion and drainage of $150 \mathrm{ml}$ of pus. Contrast medium was injected into the abscess cavity in order to confirm the catheter's position. to surgical drainage or resection; however, it might be complicated by pneumothorax, empyema, hemothorax, and bronchopleural fistula [2].

Bronchoscopic catheter drainage has emerged as an alternative to PCD, with potential advantages in selected patients, such as those with a fairly central abscess or with an airway leading to the cavity $[1,2]$. However, other than the case series of 42 patients published by Herth et al. [1] in 2005, publications regarding bronchoscopic drainage (BD) of lung abscesses have been scarce, restricted mainly to case reports and small case series [3-6].

We report herein our experience in 15 patients with lung abscesses who failed to respond to antibiotic treatment and underwent $16 \mathrm{BD}$ procedures in our center in the past decade (2006-2016), all with the use of a pigtail catheter. We describe the patient characteristics and the $\mathrm{BD}$ procedures, including technique, outcome measures, and adverse events.

\section{Methods}

This retrospective study was approved by the institutional review board at Rabin Medical Center, Israel. Data were derived from paper and electronic patient records.

\section{Patient Selection}

Patients selected for this BD procedure were hospitalized at Rabin Medical Center due to a lung abscess in the study period starting from September 2006 to January 2016, and either showed no improvement or deteriorated despite appropriate antibiotic treatment. Failure to respond to antibiotic therapy was clinically determined by the treating physicians and pulmonology consultants based on the patient's general status as well as clinical, laboratory, and imaging parameters, such as continuation of fever, increase in leukocyte count, and increase in abscess size despite treatment. A preference was set for patients with a centrally located abscess that would be accessible to BD. Other alternatives, such as PCD, were also considered.

\section{Procedure}

All BD procedures were done at the Pulmonary Institute of the Rabin Medical Center, Israel, during the study period. Flexible bronchoscopy was performed under moderate sedation through a nasal approach by an experienced endoscopist. Under fluoroscopic imaging, a guidewire was inserted into the abscess cavity through the working channel of the bronchoscope. In some cases, laser was used in order to penetrate the abscess wall and facilitate the guidewire insertion [6]. The guidewire was inserted with its stiff end leading. Once the guidewire was in place, the bronchoscope was removed. A pigtail catheter for endovascular use of $110 \mathrm{~cm}$ in length (Boston Scientific) of at least $6 \mathrm{~F}$ was passed over the guidewire into the cavity, and the wire was then removed (Fig. 1,2). The correct position of the pigtail catheter was confirmed with the injection of contrast medium (Fig. 1c). Pus from the abscess cavity was then drained through the catheter. At the end of the procedure, the catheter was secured at the nose and remained in place for a few days. It was flushed 2-4 times daily with $80-160 \mathrm{mg}$ of gentamycin [1], unless deemed unnecessary following pus culture results. At all other times, the catheter was open to gravity drainage. Following the procedure, patients continued to receive their systemic antibiotic treatment, which was later adjusted according to pus culture results. 
Fig. 2. a Chest radiograph of a 71-year-old woman (patient 9, Table 1) with a right middle lobe abscess, who failed to respond to 14 days of antibiotic treatment. b-e Sequential radiographs taken at days $0,1,2$, and 3 following bronchoscopic pigtail catheter insertion, showing gradual drainage of abscess content and decrease in abscess size. The patient improved and was discharged home 6 days following the procedure.
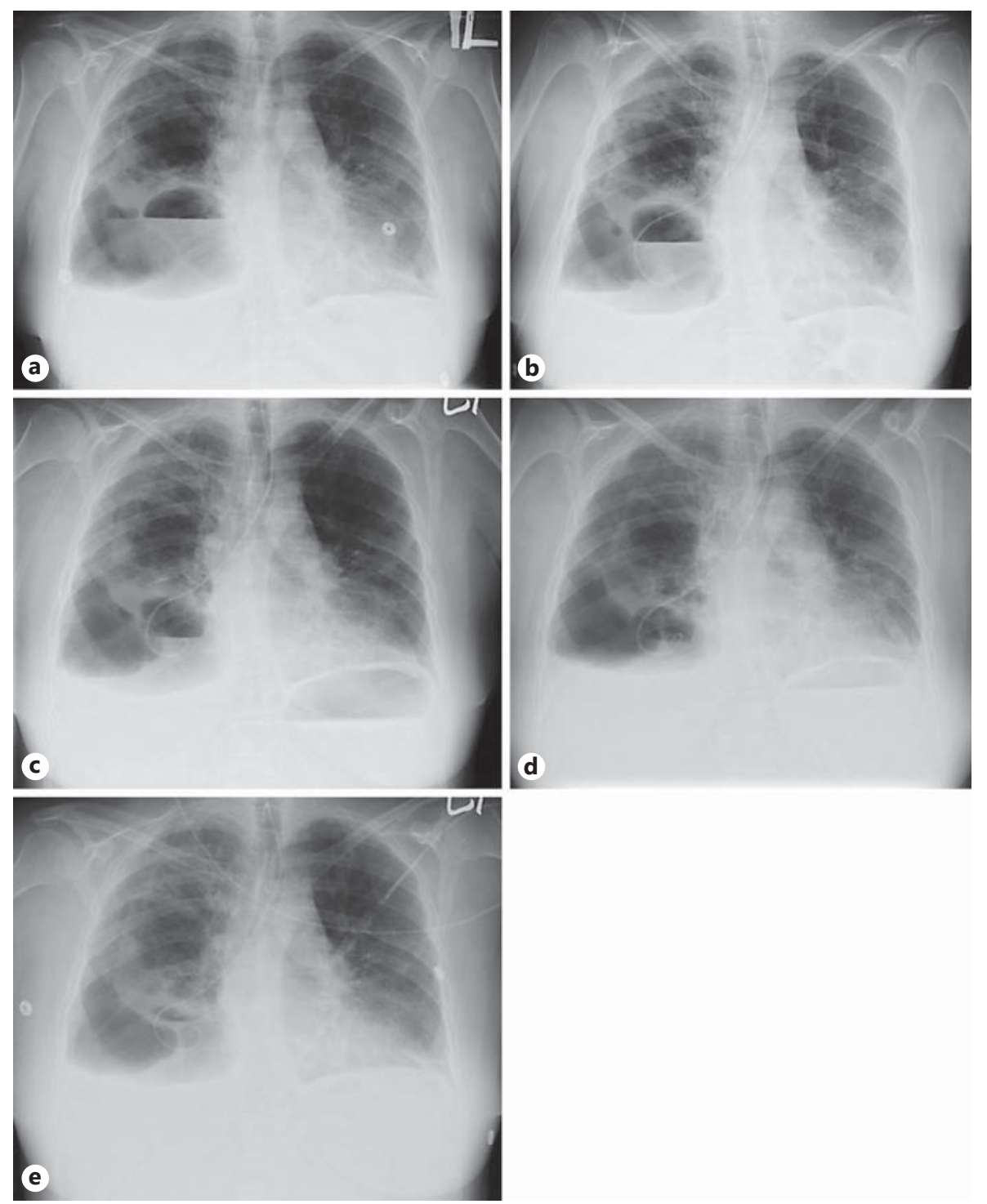

\section{Data Analysis}

Descriptive statistics were used in order to describe the characteristics of the patients and the procedures. Median values were preferred over mean ones in order to eliminate the effect of extremes.

\section{Results}

Fifteen patients ( 9 males, 6 females; median age 59 years, range 17-71) underwent $16 \mathrm{BD}$ procedures (Table 1). One patient (patient 14, Table 1) unintentionally self-extracted the pigtail catheter after less than 1 day and failed to improve, necessitating a second BD procedure
6 days later, which then led to a rapid clinical improvement.

A pigtail catheter was successfully inserted into the abscess cavity and pus drainage was achieved in 13 out of 16 procedures $(81 \%)$ conducted in 12 patients. The pigtail catheter had to be retracted in 1 of these patients, who developed a pneumothorax and empyema following its insertion, necessitating chest tube drainage. The pigtail catheter was left in place for continuous drainage in 12 procedures (75\%) in the remaining 11 patients. A pigtail catheter could not be inserted into the abscess cavity in 3 patients; in 2 patients, the abscess could not be penetrated, and in another patient, significant hemorrhage occurred during the procedure. 
Table 1. Individual patient characteristics

\begin{tabular}{|c|c|c|c|c|c|c|c|c|c|}
\hline $\begin{array}{l}\text { Patient } \\
\text { No. }\end{array}$ & $\begin{array}{l}\text { Underlying } \\
\text { disease/relevant } \\
\text { history }\end{array}$ & Sex & $\begin{array}{l}\text { Age, } \\
\text { years }\end{array}$ & $\begin{array}{l}\text { Pre-BD } \\
A B \text {, days }\end{array}$ & $\begin{array}{l}\text { Post-BD } \\
\text { AB, days }\end{array}$ & $\begin{array}{l}\text { Catheter in } \\
\text { place, days }\end{array}$ & Culture & Outcome & $\begin{array}{l}\text { Dicharge } \\
\text { post-BD, } \\
\text { days }\end{array}$ \\
\hline 2 & $\begin{array}{l}\text { pneumonia/ } \\
\text { asthma }\end{array}$ & $\mathrm{f}$ & 35 & 12 & 36 & 6 & $\begin{array}{l}\text { Peptostreptococcus } \\
\text { micros }\end{array}$ & improved & 8 \\
\hline 4 & $\begin{array}{l}\text { pneumonia/ } \\
\text { DM }\end{array}$ & $\mathrm{m}$ & 38 & 26 & 11 & 4 & Enterobacter sp. & improved & 4 \\
\hline 5 & $\begin{array}{l}\text { pneumonia/ } \\
\text { COPD }\end{array}$ & $\mathrm{f}$ & 68 & 14 & 28 & $\mathrm{NC}$ & Enterobacter sp. & $\begin{array}{l}\text { improved after } \\
\text { PCD }\end{array}$ & 6 \\
\hline 6 & $\begin{array}{l}\text { pneumonia/ } \\
\text { asthma, DM, IS }\end{array}$ & $\mathrm{f}$ & 44 & $>30$ & 22 & 5 & Pseudomonas & $\begin{array}{l}\text { did not } \\
\text { improve }^{b}\end{array}$ & $22^{\mathrm{b}}$ \\
\hline 8 & $\begin{array}{l}\text { pneumonia/ } \\
\text { BE }\end{array}$ & $\mathrm{m}$ & 47 & 13 & 30 & 5 & $\begin{array}{l}\text { Staphylococcus } \\
\text { aureus }\end{array}$ & improved & 13 \\
\hline 9 & $\begin{array}{l}\text { pneumonia/ } \\
\text { CEP, DM }\end{array}$ & $\mathrm{f}$ & 71 & 14 & 27 & 3 & negative & improved & 6 \\
\hline 10 & lung cancer & $\mathrm{m}$ & 62 & 6 & 21 & 6 & Enterococcus & improved & 7 \\
\hline 11 & $\begin{array}{l}\text { pneumonia/ } \\
\text { NF, COPD }\end{array}$ & $\mathrm{m}$ & 66 & 5 & 60 & $\mathrm{NC}$ & negative & slowly improved & 8 \\
\hline 12 & lung cancer & $\mathrm{f}$ & 59 & 7 & 14 & $4^{\mathrm{d}}$ & negative & improved & 11 \\
\hline 13 & $\begin{array}{l}\text { pneumonia/ } \\
\text { IPF }\end{array}$ & $\mathrm{m}$ & 61 & 28 & 16 & CR & $\begin{array}{l}\text { Acinetobacter, } \\
\text { Pseudomonas, } \\
\text { Klebsiella }\end{array}$ & complication $^{\mathrm{c}}$ & 23 \\
\hline 14 & $\begin{array}{l}\text { pneumonia/ } \\
\text { IPF, IS }\end{array}$ & $\mathrm{m}$ & 69 & 7 & 48 & $<1,2^{\mathrm{e}}$ & Nocardia sp. & improved & 21 \\
\hline
\end{tabular}

$\mathrm{BD}$, bronchoscopic drainage; $\mathrm{AB}$, antibiotics; NA, data not available; NC, pigtail catheter could not be inserted; CR, catheter inserted and then retracted; DM, diabetes mellitus; COPD, chronic obstructive pulmonary disease; IS, immunosuppression; CEP, chronic eosinophilic pneumonia; NF, neurofibromatosis type I; BE, bronchiectasis; IPF, idiopathic pulmonary fibrosis; PCD, percutaneous catheter drainage. ${ }^{a}$ Pulmonary hemorrhage that required surgical intervention. ${ }^{\mathrm{b}}$ This patient, a severe asthmatic and diabetic on chronic oral corticosteroid treatment, did not improve despite successful BD. She subsequently had PCD inserted, developed multiple bilateral abscesses and neutropenia, and eventually died 22 days later. ${ }^{c}$ Pneumothorax and empyema, necessitating chest tube insertion. The pigtail catheter had to be retracted in light of this complication. ${ }^{\mathrm{d}}$ Pigtail catheter unintentionally extracted after 4 days. The patient improved. ${ }^{\mathrm{e}}$ This patient unintentionally extracted the pigtail catheter $<1$ day following the procedure and failed to improve, requiring a second BD procedure.

Of the 11 patients who remained with a pigtail catheter, 10 improved (91\%), as was determined by resolution of fever and leukocytosis; resolution of fever occurred at a median of 2 days following the procedure (range $<1-4$; data available for 8 out of 10 patients), and patients were discharged from hospital after a median of 8 days (range 4-21). One patient (patient 6, Table 1), a severe asthmatic and diabetic on chronic oral corticosteroid treatment, did not improve despite successful BD, subsequently had
PCD inserted, developed multiple bilateral abscesses and neutropenia, and eventually died 3 weeks later.

The indication for all BD procedures was lack of response to antibiotic treatment. Median antibiotic treatment duration was 14 days (range 5 to $>30$ ) before the procedure, and 22 days (range 11-60) after the procedure. In the vast majority of patients, the pigtail catheter was flushed 2-4 times daily with gentamycin. The catheter was extracted after a median of 4 days (range 2-6), 
except in 2 patients who unintentionally self-extracted the catheter after 4 and $<1$ days, respectively; the former improved, while the latter did not and required a second BD.

Procedure-related complications occurred in 2 out of 16 procedures $(13 \%)$. One patient (patient 13 , Table 1 ) had pneumothorax and empyema, necessitating pigtail catheter retraction and chest tube insertion. Of note, the peripheral location of the abscess was relatively adjacent to the pleura. The patient eventually improved, fever resolved after 9 days, and the patient was discharges 23 days following the procedure. The second patient (patient 3 , Table 1) presented with pulmonary hemorrhage which necessitated surgical intervention. Pathology revealed intralobar sequestration with abscess formation, and the patient eventually recovered.

\section{Discussion}

Most lung abscesses are successfully treated with systemic antibiotics and postural drainage to increase drainage through the adjacent airways [2,7]. However, some patients fail to respond and require invasive abscess drainage $[1,2,8]$. In the past decades, $\mathrm{BD}$ of lung abscesses has emerged as an alternative to percutaneous computed tomography-guided drainage (PCD) or surgery [1], although only limited data have been reported in the literature [2].

Our report of $16 \mathrm{BD}$ procedures adds to the existing literature. Successful BD was achieved in 13 out of 16 procedures $(81 \%)$, leading to rapid improvement in the majority of patients. This figure is comparable to the success rate of PCD (84\%) in a recent review of lung abscess drainage [2]. The fairly low complication rate of $13 \%$ is also comparable to that of PCD (16\%) [2].

Most patients in this case series were of advanced age, with significant comorbidities, such as lung diseases, lung cancer, diabetes mellitus, and immunosuppression. It is probable that such patients are at a higher risk to fail antibiotic treatment and require a drainage procedure [9]. In 4 patients (27\%), attempts to culture the causative organism were not successful in sputum, bronchoalveolar lavage, and/or abscess aspirate. This negative culture rate is consistent with previous reports $[10,11]$ and may potentially be improved using molecular bacterial detection methods [11].

The optimal duration of catheter drainage is currently unknown. In the series by Herth et al. [1], the mean drainage duration was 6.2 days (range 3-21), substantially lon-

Bronchoscopic Drainage of Lung

Abscesses ger than that reported in our series. The time of catheter extraction in our series was guided by clinical and laboratory improvement, including resolution of fever and leukocytosis, and occurred after a median of 4 days (mean 4.3 days; range 2-6). Even with this shorter drainage duration, 10 of 11 patients improved (91\%), suggesting that this shorter clinical improvement-guided drainage duration is sufficient in most cases.

The pigtail catheter was empirically flushed with gentamycin, as was previously described by Herth et al. [1]. Although flushing the catheter with gentamycin makes clinical sense and is possibly helpful, it should be emphasized that this approach has not been tested in a randomized controlled trial. In addition, as gentamycin might be systemically absorbed from the lungs [12], caution should be taken and serum drug levels may need to be monitored in patients with renal failure or those already receiving systemic gentamycin.

Two patients unintentionally self-extracted the pigtail catheter ahead of time; one of them did not improve as a result and required a repeated procedure. It is, therefore, imperative to appropriately fixate the pigtail catheter at the nose and possibly at a second location on the face in order to avoid premature displacement of the catheter.

In contrast to the report by Herth at al. [1], we did not limit the procedure only to patients with an airway leading to the abscess, and in some cases even used laser in order to penetrate the abscess wall [6]. This approach enabled some patients without a leading airway to benefit from the procedure; however, due to the possible higher risk entailed, this approach should be considered on a case by case basis.

Massive aspiration due to endobronchial spillage of abscess contents was previously described following bronchoscopy in a lung abscess patient [13]; however, neither in the report by Herth et al. [1] nor in our experience has this complication occurred. Effective drainage of the abscess contents via the pigtail catheter during and after the procedure clearly reduces this risk; although some spillage may still occur around the catheter, it is unlikely to result in massive aspiration.

Alternative treatment modalities for pulmonary abscesses that fail to respond to antibiotic therapy include PCD and surgical drainage or resection. PCD is an effective and less invasive alternative to surgical intervention and is thus preferable to surgery in most cases $[2,8,14-$ 16]. However, it might be complicated by pneumothorax, empyema, hemothorax, and bronchopleural fistula $[2,8]$. Before considering PCD for refractory lung abscesses, it is important to perform bronchoscopy in order to rule 
out bronchial obstruction and/or malignancy, which are indications for surgical resection [2]. VanSonnenberg et al. [8] reported a case of hemothorax, when the catheter traversed normal lung en route to the abscess, and suggested that PCD is preferable in more peripheral abscesses in which the catheter route runs through an abscesspleural syndesis.

Compared to PCD, less experience has been accumulated with BD. Data from the current report, as well as from the report by Herth et al. [1], suggest that BD has an efficacy and safety profile that is comparable to that reported for PCD, although no direct comparison was executed. Herth et al. [1] reported no procedure-related complications in a series of 42 patients other than 2 patients requiring mechanical ventilation for $24 \mathrm{~h}$ after the procedure. In our series, procedure-related complications occurred in 2 out of 16 procedures, namely 1 case of pulmonary hemorrhage and 1 case of pneumothorax and empyema, the latter in a patient with a peripheral abscess, relatively adjacent to the pleura. In summary, the evidence suggests that $\mathrm{BD}$ can be considered an acceptable alternative to PCD in selected patients, especially if there is an airway leading to the abscess cavity. Moreover, we consider $\mathrm{BD}$ a preferred option for centrally located abscesses, in which a PCD catheter would have to traverse a significant amount of healthy lung tissue.

This study has several strengths as well as some limitations. Although Rabin Medical Center is a relatively large center (about 1,300 hospital beds), within the study period of a decade only 15 patients were considered eligible for the BD procedure. We believe that our experience reflects the real-life situation in most hospitals, where lung abscesses that require invasive intervention are not very common, thus making it hard to gain experience in performing this type of procedure. It was, therefore, reassuring to find that, despite this shortcoming, the procedure was successful in the majority of patients, while maintain- ing a relatively low complication rate comparable to previously reported rates regarding PCD [2]. In our opinion, this real-life experience should encourage other medical centers to consider this procedure as an option in appropriate cases. The most significant limitation of the study is its retrospective methodology. Despite our best efforts to retrieve complete data from the medical records, some data were unavailable in 2 patients (Table 1), but it is unlikely that these would affect our conclusions. Finally, patients were selected according to the best clinical judgement of their treating physicians and pulmonology consultants rather than according to a prospective set of rules used by a multidisciplinary committee.

In conclusion, this work adds to the existing literature in establishing BD as an acceptable alternative to percutaneous drainage in selected lung abscess patients in whom conventional antibiotic therapy fails. It is appropriate in those patients with an airway obstruction or a fairly central abscess, especially if a leading airway can be demonstrated. Future research should focus on devising and validating patient selection criteria for both bronchoscopic and percutaneous lung abscess draining procedures.

\section{Acknowledgements}

The authors express their gratitude to Ms. Dalia Dawn Orkin for her important English language contributions and editing services.

\section{Financial Disclosure and Conflicts of Interest}

No financial support or other benefits were received from commercial sources for this work, and there are no conflicts of interest that any of the authors may have with regard to this work.

\section{References}

1 Herth F, Ernst A, Becker HD: Endoscopic drainage of lung abscess. Chest 2005;127: 1378-1381.

2 Wali SO: An update on the drainage of pyogenic lung abscesses. Ann Thorac Med 2012; $7: 3-7$.

3 Connors JP, Roper CL, Ferguson TB: Transbronchial catheterization of pulmonary abscess. Ann Thorac Surg 1975; 19:254-260.

4 Rowe LD, Keane WM, Jafek BW, Atkins JP Jr: Transbronchial drainage of pulmonary abscesses with the flexible fiberoptic bronchoscope. Laryngoscope 1979;89:122-128.
5 Schmitt GS, Ohar JM, Kanter KR, Naunheim KS: Indwelling transbronchial catheter drainage of pulmonary abscess. Ann Thorac Surg 1988;45:43-47.

6 Shlomi D, Kramer MR, Fuks L, Peled N, Shitrit D: Endobronchial drainage of lung abscess: the use of laser. Scand J Infect Dis 2010; 42:65-68.

7 Kuhajda I, Zarogoulidis K, Tsirgogianni K, Tsavlis D, Kioumis I, Kosmidis C, Tsakiridis K, Mpakas A, Zarogoulidis P, Zissimopoulos A, Baloukas D, Kuhajda D: Lung abscess-etiology, diagnostic and treatment options. Ann Transl Med 2015;3:183. 
8 vanSonnenberg E, D'Agostino HB, Casola G, Wittich GR, Varney RR, Harker C: Lung abscess: CT-guided drainage. Radiology 1991; 178:347-351.

9 Mwandumba HC, Beeching NJ: Pyogenic lung infections: factors for predicting clinical outcome of lung abscess and thoracic empyema. Curr Opin Pulm Med 2000;6:234-239.

10 Hirshberg B, Sklair-Levi M, Nir-Paz R, BenSira L, Krivoruk V, Kramer MR: Factors predicting mortality of patients with lung abscess. Chest 1999;115:746-750.
11 Mukae H, Noguchi S, Naito K, Kawanami T, Yamasaki K, Fukuda K, et al: The importance of obligate anaerobes and the Streptococcus anginosus group in pulmonary abscess: a clone library analysis using bronchoalveolar lavage fluid. Respiration 2016;92:80-89.

12 Lake KB, Dyke JJ, Rumsfeld JA: Combined topical pulmonary and systemic gentamicin: the question of safety. Chest 1975;68:62-64.

13 Hammer DL, Aranda CP, Galati V, Adams FV: Massive intrabronchial aspiration of contents of pulmonary abscess after fiberoptic bronchoscopy. Chest 1978;74:306-307.
14 Yellin A, Yellin EO, Lieberman Y: Percutaneous tube drainage: the treatment of choice for refractory lung abscess. Ann Thorac Surg 1985;39:266-270.

15 Wali SO, Shugaeri A, Samman YS, Abdelaziz M: Percutaneous drainage of pyogenic lung abscess. Scand J Infect Dis 2002;34:673-679.

16 Parker LA, Melton JW, Delany DJ, Yankaskas BC: Percutaneous small bore catheter drainage in the management of lung abscesses. Chest 1987;92:213-218. 